\title{
Higher order quark-mesonic scattering processes and the phase structure of QCD* $^{*}$
}

\author{
J. M. Pawlowski ${ }^{1,2}$ and F. Rennecke $e^{1,2}$ \\ ${ }^{1}$ Universität Heidelberg, Germany; ${ }^{2}$ GSI, Darmstadt, Germany
}

We study the chiral phase transition of two-flavor quantum chromodynamics (QCD) at finite temperature $T$ and quark chemical potential $\mu$ [1]. At not too large chemical potential, the chiral dynamics in the vicinity of the phase boundary are driven by the lightest hadronic states, the pions and the sigma meson. Thus, in order to arrive at a quantitative picture of the matter sector of QCD, these mesonic degrees of freedom need to be taken into account accurately. We therefore employ a linear quark-meson model which captures spontaneous chiral symmetry breaking $S U\left(N_{f}\right)_{L} \otimes S U\left(N_{f}\right)_{R} \rightarrow S U\left(N_{f}\right)_{V}$. Quantum fluctuations are included by means of the functional renormalization group. The scale dependent effective action reads [2]:

$$
\begin{aligned}
\Gamma_{k}= & \int_{x}\left\{i Z_{\psi, k} \bar{\psi}\left(\gamma_{\mu} \partial_{\mu}+\gamma_{0} \mu\right) \psi+\frac{1}{2} Z_{\phi, k}\left(\partial_{\mu} \phi\right)^{2}\right. \\
& \left.+V_{k}(\rho)-c \sigma+h_{k}(\rho) \bar{\psi}\left(\gamma_{5} \vec{\tau} \vec{\pi}+i \sigma\right) \psi\right\}
\end{aligned}
$$

where $\rho=\phi^{2} / 2=\vec{\pi}^{2}+\sigma^{2}$. We systematically study the effect of higher order multi-meson as well as quarkantiquark multi-meson scattering processes on the chiral phase structure of QCD by expanding the effective potential $V_{k}(\rho)$ and the field-dependent Yukawa coupling $h_{k}(\rho)$ in powers of $\rho$. This corresponds to an expansion of the effective action $\Gamma_{k}$ in terms of $n$-point functions. We observe that these higher order operators play a quantitatively important role for the chiral phase transition. Furthermore, the expansions of both, the effective potential and the Yukawa coupling, converge rapidly. This implies that we have good control over the quantitative precision of our results. For the effect of different parts of the truncation (1) on the phase boundary, see Fig. 1.

As a result of the explicit chiral symmetry breaking $-c \sigma$, which is directly related to finite current quark masses, we observe a crossover phase transition. The transition temperature/chemical potential in this case is not uniquely defined and we therefore compare different definitions of the phase boundary. Fig. 2 shows the resulting phase diagram. We find a crossover transition for $\mu<291 \mathrm{MeV}$. The large deviations in the transition temperatures between different definitions of the phase boundary indicate a very broad crossover. We note that the same is true for the curvature of the phase boundary at vanishing density. The crossover transition gets steeper towards the critical endpoint which we find at $\left(T_{c}, \mu_{c}\right)=(50,291) \mathrm{MeV}$.

* Work supported by Helmholtz Alliance HA216/EMMI and by ERCAdG-290623
For the future this analysis can be used as a starting point for studies aiming towards full QCD, including baryonic degrees of freedom as well as the gauge sector of QCD.

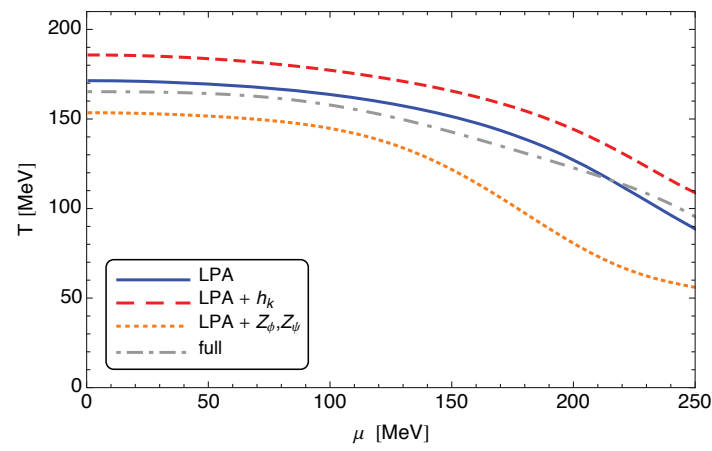

Figure 1: The crossover phase boundary for different truncations. LPA denotes the quark-meson model with only a running effective potential. The dot-dashed curve shows the full result of (1).

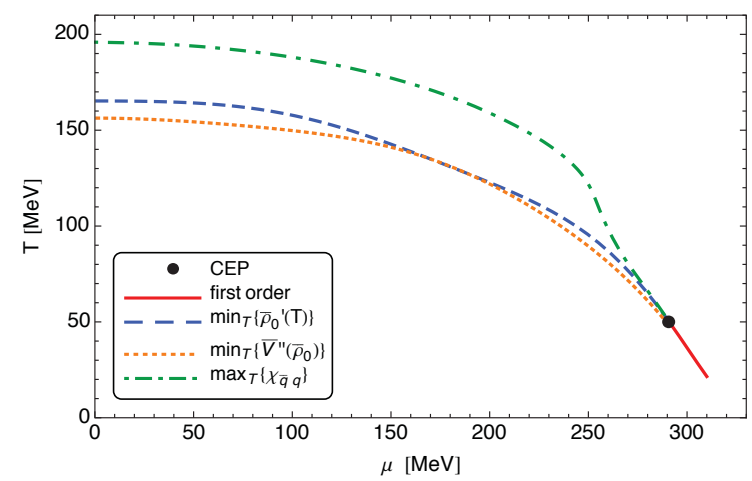

Figure 2: The phase diagram of the chiral transition of QCD. The crossover transition was extracted from three different quantities: the minimum of the effective potential (dashed), the quartic meson coupling (dotted) and the chiral susceptibility (dot-dashed).

\section{References}

[1] P. Braun-Munzinger and J. Wambach, "Colloquium: Phase diagram of strongly interacting matter", Rev. Mod. Phys. 81, p. 1031 (2009)

[2] J. M. Pawlowski and F. Rennecke, "Higher order quarkmesonic scattering processes and the phase structure of QCD”, hep-ph/1403.1179 (2014) 